\title{
O que os produtores de goiaba (Psidium guajava L.) do Alto Sertão sergipano pensam a respeito das aves silvestres?
}

What do guava producers (Psidium guajava L.) from the Alto Sertão region, state of Sergipe, think about wild birds?

\author{
C. Silva ${ }^{1 *}$ J. Ruiz-Esparza ${ }^{2} ;$ F. O. Silva ${ }^{2,3} ;$ A. S. Ribeiro ${ }^{4}$ \\ ${ }^{1}$ Programa de Pós-Graduação em Desenvolvimento e Meio Ambiente, Universidade Federal de Sergipe, 49100-000, \\ São Cristóvão-SE, Brasil \\ ${ }^{2}$ Núcleo em Educação e Ciências Agrárias e da Terra, Universidade Federal de Sergipe, 49680-000, Nossa Senhora da \\ Glória-SE, Brasil \\ ${ }^{3}$ Instituto Nacional de Ciência e Tecnologia em Estudos Interdisciplinares e Transdisciplinares em Ecologia e \\ Evolução (INCT IN-TREE) \\ ${ }^{4}$ Departamento de Ecologia, Universidade Federal de Sergipe, 49100-000, São Cristóvão-SE, Brasil
}

*silvac.bio@gmail.com

(Recebido em 16 de março de 2020; aceito em 11 de agosto de 2020)

\begin{abstract}
A presença de aves em agroecossistemas pode aumentar significativamente a produção agrícola e os valores de marcado, por meio, especialmente, do controle de pragas, importante serviço ecossistêmico, mas que não costuma ser percebido pelos agricultores. Deste modo, esta pesquisa buscou investigar e compreender a percepção dos produtores de goiaba (Psidium guajava L.) do Alto Sertão sergipano sobre a avifauna silvestre local, a fim contribuir para a conservação da avifauna e para o desenvolvimento de práticas agrícolas mais sustentáveis. Os dados foram coletados de julho de 2017 a março de 2018, por meio de entrevistas estruturadas com 85 produtores de goiaba. Foi citado um total de 50 espécies de aves pelos entrevistados. Cerca de $70 \%$ dos produtores entrevistados afirmaram que as aves visitam as plantações para se alimentar das goiabas, causando prejuízos econômicos. Poucos produtores percebem as aves como elementos importantes dentro do sistema agrícola, realizando poucas práticas para sua proteção. Assim, faz-se necessário estimular a percepção positiva dos produtores sobre a interação das aves com o ambiente agrícola, uma vez que esses animais podem fornecer importantes serviços ecossistêmicos (ex., controle de pragas).

Palavras-chave: agricultura sustentável, avifauna, serviços ecossistêmicos.
\end{abstract}

The presence of birds in the agro-ecosystems can significantly increase crop production and market values, especially through pest control, an important ecosystem service which is not usually perceived by farmers. Our research sought to investigate and understand the perception of guava producers (Psidium guajava L.) from the Alto Sertão region, state of Sergipe, about the native birds, in order to generate knowledge for their conservation and the development of a more sustainable agriculture. Data were collected from July 2017 to March 2018, through structured interviews with 85 guava producers. A total of 50 bird species were cited by respondents. About $70 \%$ of the interviewed producers stated that birds visit the plantations to feed on guavas, causing economic losses. A small number of guava roducers perceive birds as important elements within the agricultural system, carrying out few practices for their protection. Thus, it is necessary to stimulate a positive perception of producers about the interaction of birds with the agricultural environment, since these animals can provide important ecosystem services (eg. pest control).

Keywords: sustainable agriculture, avifauna, ecosystem services.

\section{INTRODUÇÃO}

Em culturas agrícolas, algumas espécies de aves não costumam ser apreciadas por agricultores, em função dos prejuízos econômicos causados por esses animais [1, 2, 3, 4]. Algumas espécies causam danos diretos por granivoria e frugivoria [5], ou indiretos, pelo consumo de polinizadores [6] e inimigos naturais de pragas [7]. Desta forma, os agricultores adotam ações para eliminar as aves de suas lavouras que vão desde a captura ao abate por armas de fogo e aplicação de agrotóxicos nas sementes [1,2].

Em fruticultura, algumas espécies de aves e insetos-praga, são os principais responsáveis pela diminuição da produção $[8,9]$. Produtores rurais, em Minas Gerais, relataram que culturas frutíferas (ex., abacate, acerola, ameixa, amora, banana, frutas cítricas, goiaba, jabuticaba, mamão e manga) 
são as mais danificadas por aves [10]. Nos Estados Unidos, produtores de cereja relataram que os danos causados por aves é um dos principais fatores que afetam seus lucros, ou o principal fator [11]. Produtores de maçã e uva perdem dezenas de milhões de dólares a cada ano, devido à predação dos frutos por aves e despesas com medidas de manejo para evitar ou mitigar os danos [11]. Segundo Dellamano (2006) [12], as aves reduzem o rendimento das colheitas consumindo e danificando os frutos, deixando-os susceptíveis a patógenos e exigindo que os frutos sejam colhidos antes do amadurecimento, resultando em produtos economicamente inferiores.

Em contrapartida, a presença de aves em agroecossistemas também pode aumentar significativamente a produção agrícola e os valores de mercado, por meio, especialmente, do controle de pragas [13, 14], um importante serviço ecossistêmico, mas que não costuma ser percebido pelos agricultores [3, 15, 16, 17]. Aves que se alimentam de insetos nocivos e outras pragas do ambiente agrícola podem aumentar significativamente o rendimento das culturas, beneficiando os agricultores [18, 19, 20]. Kellermann et al. (2008) [21], em um estudo na Jamaica, verificaram que as aves reduziam de $1 \%$ a $14 \%$ os danos causados pela broca-do-café (Hypothenemus hampei Ferrari, 1867; Coleoptera: Scolytidae) ao consumirem esses insetos, aumentando, consequentemente, o valor de mercado do café em até US\$ 105/ha. Da mesma forma, Mols e Visser (2007) [22] relataram que o chapim-real (Parus major Linnaeus, 1758; Passeriformes: Paridae) reduzia os danos causados por lagartas em pomares de maçã em até 50\%, em comparação com pomares sem sua presença.

O conhecimento e a percepção dos agricultores sobre a avifauna silvestre pode interferir nas atitudes e ações que cotidianamente estabelecem com este grupo animal, com vistas a atrair, repelir, controlar e/ou eliminar espécies de seus plantios [3]. Assim, compreender o conhecimento e a opinião dos agricultores sobre as aves que ocorrem em seus cultivos contribuirá para o desenvolvimento de ações educativas e de conservação da avifauna [23, 24]. Deste modo, esta pesquisa teve por objetivo investigar a percepção dos produtores de goiaba (Psidium guajava L.) do Alto Sertão sergipano sobre a avifauna silvestre e sua interação com os pomares de goiaba. A escolha de trabalhar com pomares de goiaba se deu pela posição de destaque que o cultivo da goiaba ocupa hoje na área estudada. A cultura da goiaba tem se destacado na região como uma das principais culturas de maior rentabilidade e possibilidade de expansão [25]. Em 2017, a produção de goiaba correspondeu a 8.480 toneladas, com produtividade média de $19.953 \mathrm{~kg} / \mathrm{ha}$ [26].

\section{MATERIAL E MÉTODOS}

\section{1. Área de estudo}

Este estudo foi conduzido em 39 pomares de goiaba, nos assentamentos agrícolas Califórnia e Jacaré-Curituba, localizados no território do Alto Sertão no Noroeste do estado de Sergipe, abrangendo os municípios de Canindé de São Francisco (09³8’31'S, 3747'16”O) e Poço

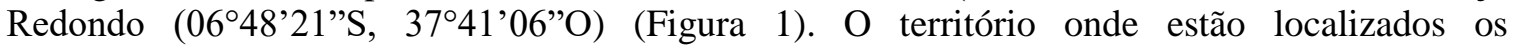
assentamentos apresenta características climáticas, segundo a classificação Köppen, do tipo Bsh, semiárido seco e quente, caracterizado por escassez de chuvas com grande irregularidade em sua distribuição, índices elevados de evaporação e temperatura média acima de $25^{\circ} \mathrm{C}[27,28]$.

Nos assentamentos Califórnia e Jacaré-Curituba são desenvolvidas atividades do setor primário da economia, como a agricultura de policultivos e pecuária. Dentre os cultivos produzidos, destacam-se as leguminosas, hortaliças e frutíferas, sendo o quiabo, a macaxeira, o milho, o feijão, a acerola e a goiaba as principais culturas exploradas pelos produtores [25]. 

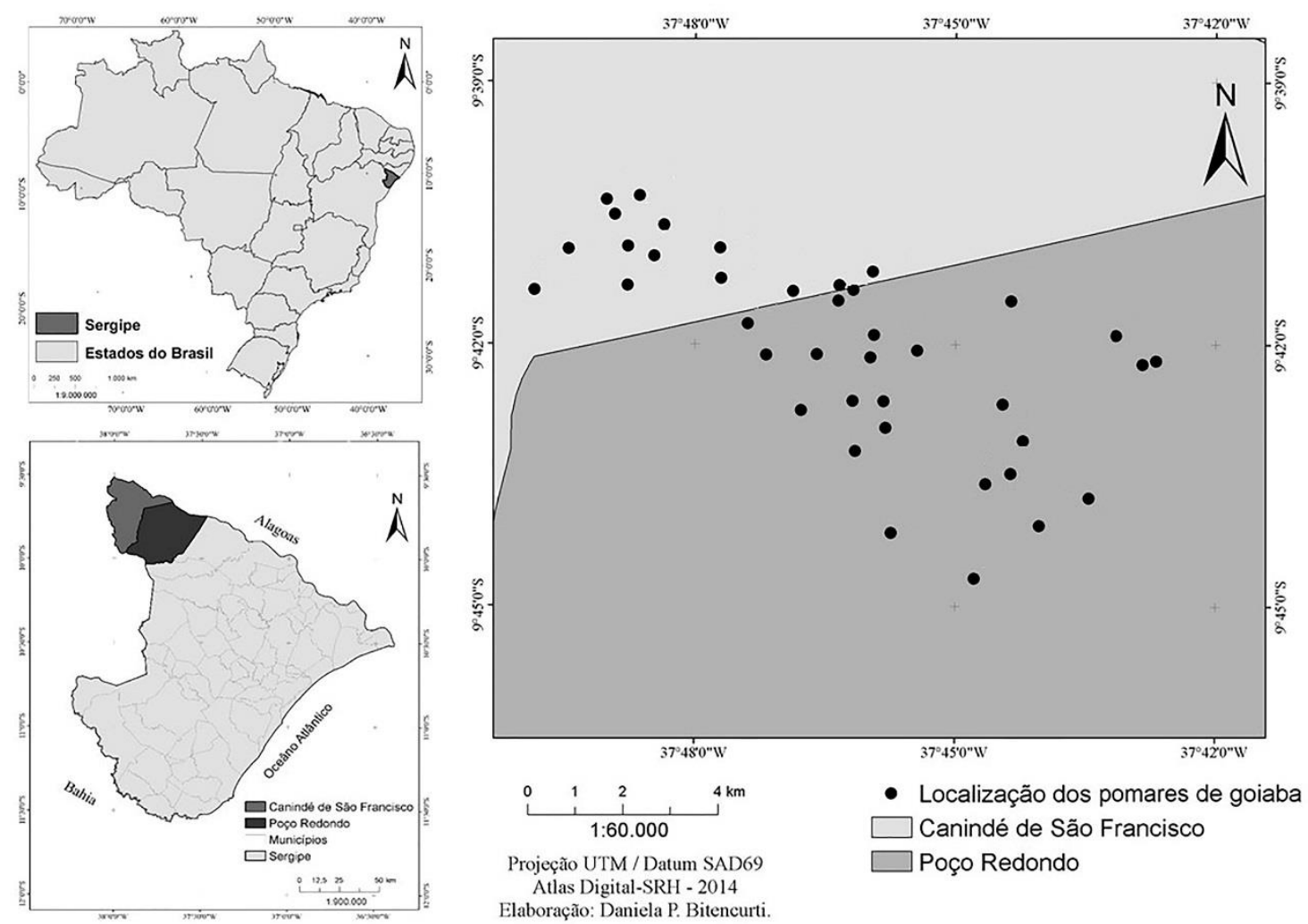

Figura 1: Localização dos 39 pomares de goiaba amostrados nos assentamentos agrícolas Califórnia e Jacaré-Curituba (Canindé de São Francisco e Poço Redondo, SE).

\subsection{Coleta de dados}

Os dados relativos ao conhecimento e percepção dos produtores de goiaba sobre as aves foram obtidos mediante a realização de entrevistas estruturadas $[29,30]$ realizadas entre os meses de julho de 2017 e março de 2018 por produtores de 39 propriedades. As perguntas do questionário (Tabela 1) foram respondidas voluntariamente por 85 informantes. Em média, foram entrevistados dois trabalhadores de cada propriedade. Os informantes receberam explicações sobre os objetivos e implicações da pesquisa contidas no Termo de Consentimento Livre e Esclarecido, atendendo à Resolução 466/12 do Conselho Nacional de Saúde por meio do Comitê de Ética em Pesquisa [31]. Após a assinatura do termo, uma via original foi fornecida ao informante e a outra ao pesquisador. Esta pesquisa foi aprovada e autorizada pelo Comitê de Ética em Pesquisa com seres humanos da Universidade Federal de Sergipe (CAAE 99311118.2.0000.5546).

Tabela 1: Perguntas respondidas pelos produtores de goiaba dos assentamentos agrícolas Califórnia e Jacaré-Curituba (Canindé de São Francisco e Poço Redondo, SE) durante as entrevistas.

\begin{tabular}{|c|c|}
\hline Número & Pergunta \\
\hline 1 & As aves visitam as goiabeiras? O que elas fazem nas goiabeiras? \\
\hline 2 & Quais espécies de aves você conhece? \\
\hline 3 & Você gosta de aves? Por quê? \\
\hline 4 & Você acha que as aves são importantes para a agricultura? Por quêe? \\
\hline 5 & Você acha que as aves precisam ser protegidas? Por quê?? \\
\hline 6 & Você faz algo para proteger as aves? O que? \\
\hline
\end{tabular}

As respostas dos entrevistados foram redigidas pelo próprio pesquisador, sem interferência do mesmo sobre as respostas. Procurou-se adequar a linguagem do instrumento de pesquisa a cada entrevistado para facilitar o entendimento sobre as perguntas.

A identificação das aves foi realizada através da técnica checklist/entrevista [32], com a utilização de recursos sonoros [33] e fotográficos [34] das espécies de aves constantes na área 
estudada. As espécies citadas pelos participantes foram listadas com o nome conhecido localmente e também com o nome científico, conforme a lista mais recente do Comitê Brasileiro de Registros Ornitológicos [35].

\section{RESULTADOS E DISCUSSÃO}

Foram citadas 50 espécies de aves pertencentes a 25 famílias (Tabela 2), das quais as mais representativas foram Thraupidae $(n=9)$, Columbidae $(n=7)$ e Tinamidae e Icteridae (ambas $n=$ 3 ). As espécies de aves que obtiveram maior número de citações foram, Columbina talpacoti $(\mathrm{n}=$ $83,97 \%)$, Columbina picui $(\mathrm{n}=55,65 \%)$ e Crotophaga ani $(\mathrm{n}=52,61 \%)$ (Figura 2$)$, as quais estão entre as espécies mais comuns e abundantes nos 39 pomares de goiaba amostrados [36].

Tabela 2: Espécies de aves citadas pelos produtores de goiaba dos assentamentos agrícolas Califórnia e Jacaré-Curituba (Canindé de São Francisco e Poço Redondo, SE).

\begin{tabular}{|c|c|c|}
\hline Família / Espécie & Nome local & $\begin{array}{l}\text { Número de } \\
\text { citações }\end{array}$ \\
\hline \multicolumn{3}{|l|}{ Tinamidae Gray, 1840} \\
\hline Crypturellus parvirostris (Wagler, 1827) & Nambu & 36 \\
\hline Nothura boraquira (Spix, 1825) & Codorniz & 6 \\
\hline Nothura maculosa (Temminck, 1815) & Codorna & 20 \\
\hline \multicolumn{3}{|l|}{ Ardeidae Leach, 1820} \\
\hline Bubulcus ibis (Linnaeus, 1758) & Garça & 15 \\
\hline Ardea alba (Linnaeus, 1758) & Garça-grande & 2 \\
\hline \multicolumn{3}{|l|}{ Cathartidae Lafresnaye, 1839} \\
\hline Coragyps atratus (Bechstein, 1793) & Urubu & 7 \\
\hline \multicolumn{3}{|l|}{ Accipitridae Vigors, 1824} \\
\hline Espécie não identificada & Gavião & 6 \\
\hline \multicolumn{3}{|l|}{ Rallidae Rafinesque, 1815} \\
\hline Aramides cajaneus (Statius Muller, 1776) & Três-pote & 1 \\
\hline Gallinula galeata (Lichtenstein, 1818) & Galinha-d'água & 2 \\
\hline \multicolumn{3}{|l|}{ Charadriidae Leach, 1820} \\
\hline Vanellus chilensis (Molina, 1782) & Espanta-boiada, Quero-quero & 3 \\
\hline \multicolumn{3}{|l|}{ Picidae Leach, 1820} \\
\hline Colaptes melanochloros (Gmelin, 1788) & Pica-pau & 3 \\
\hline \multicolumn{3}{|l|}{ Cariamidae Bonaparte, 1850} \\
\hline Cariama cristata (Linnaeus, 1766$)$ & Sariema & 2 \\
\hline \multicolumn{3}{|l|}{ Falconidae Leach, 1820} \\
\hline Caracara plancus (Miller, 1777) & Carcará & 5 \\
\hline \multicolumn{3}{|l|}{ Columbidae Leach, 1820} \\
\hline Columbina minuta (Linnaeus, 1766) & Rolinha-miúda, Rolinha-pivó & 5 \\
\hline Columbina picui (Temminck, 1813) & Rolinha-branca & 55 \\
\hline Columbina squammata (Lesson, 1831) & Rolinha-fogo-pagou & 3 \\
\hline Columbina talpacoti (Temminck, 1811) & $\begin{array}{c}\text { Rolinha vermelha, Rolinha-caldo-de- } \\
\text { feijão }\end{array}$ & 83 \\
\hline Leptotila sp. & Juriti & 4 \\
\hline Patagioenas picazuro (Temminck, 1813) & Asa-branca & 3 \\
\hline Zenaida auriculata (Des Murs, 1847) & Arribação, cardinheira & 6 \\
\hline \multicolumn{3}{|l|}{ Cuculidae Leach, 1820} \\
\hline Crotophaga ani (Linnaeus, 1758) & Anum, anum-preto & 52 \\
\hline Guira guira (Gmelin, 1788) & Anum-branco & 10 \\
\hline \multicolumn{3}{|l|}{ Psittacidae Rafinesque, 1815} \\
\hline Eupsittula cactorum (Kuhl, 1820) & Arara, Ararinha, Papagaio, Maritaca & 38 \\
\hline Forpus xanthopterygius (Spix, 1824) & Periquito & 15 \\
\hline \multicolumn{3}{|l|}{ Strigidae Leach, 1820} \\
\hline Athene cunicularia (Molina, 1782) & Coruja & 4 \\
\hline
\end{tabular}


Trochilidae Vigors, 1825

Espécie não identificada

Eupetomena macroura (Gmelin, 1788)

Furnariidae Gray, 1840

Pseudoseisura cristata (Spix, 1824)

Furnarius rufus (Gmelin, 1788)

Tyrannidae Vigors, 1825

Pitangus sulphuratus (Linnaeus, 1766)

Fluvicola nengeta (Linnaeus, 1766)

Corvidae Leach, 1820

Cyanocorax cyanopogon (Wied, 1821)

Troglodytidae Swainson, 1831

Troglodytes musculus (Naumann, 1823)

Turdidae Rafinesque, 1815

Turdus rufiventris (Vieillot, 1818)

Mimidae Bonaparte, 1853

Mimus saturninus (Lichtenstein, 1823)

Thraupidae Cabanis, 1847

Paroaria dominicana (Linnaeus, 1758)

Tangara sayaca (Linnaeus, 1766)

Coereba flaveola (Linnaeus, 1758)

Sporophila albogularis (Spix, 1825)

Sporophila lineola (Linnaeus, 1758)

Sporophila nigricollis (Vieillot, 1823)

Volatinia jacarina (Linnaeus, 1766)

Corysphopingus pileatus (Wied, 1821)

Sicalis flaveola (Linnaeus, 1766)

\section{Cardinalidae Ridgway, 1901}

Cyanoloxia brissonii (Lichtenstein, 1823)

\section{Icteridae Vigors, 1825}

Chrysomus ruficapillus (Vieillot, 1819)

Gnorimopsar chopi (Vieillot, 1819)

Icterus jamacaii (Gmelin, 1788)

Fringillidae Leach, 1820

Spinus yarrellii (Audubon, 1839)

Passeridae Rafinesque, 1815

Passer domesticus (Linnaeus, 1758)
Beija-flor 7

Beija-flor-de-rabo-grande 1

Casaco-de-couro 18

João-de-barro, Maria-barreira 31

Bem-te-vi 35

Lavandeira 7

Cancão 3

Garrincha 14

Sabiá, Sabiá-coca 37

Sabiá-branca 33

Galo-de-campina, Cabeça $\quad 48$

Assanhaçu 48

Cebinho 1

Viuvinho, Coleira, Coleirinha 35

Bigodinho 1

Papa-capim 3

Tiziu 9

Maria-fita 1

Canário, Canarinho, Canário-da-terra 7

Azulão 5

Brió 14

Passo-preto 9

Sofrê 14

Pinta-silva 3

Pardal 9

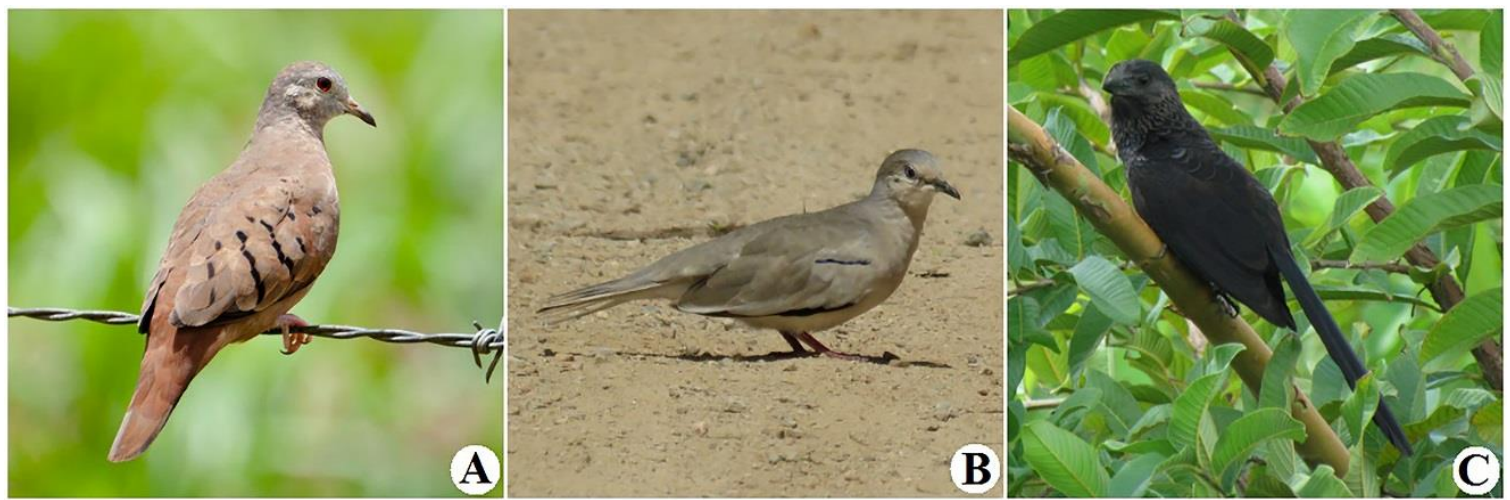

Figura 2: Espécies de aves mais citadas pelos produtores de goiaba dos assentamentos agrícolas Califórnia e Jacaré-Curituba (Canindé de São Francisco e Poço Redondo, SE). A - Columbina talpacoti, B - Columbina picui, C-Crotophaga ani. Fotos: Cleverton da Silva.

Os produtores de goiaba demonstraram conhecer poucas espécies de aves da região. O número de aves conhecidas pelos produtores entrevistados corresponde a apenas 23,3\% do total de 197 
espécies já registradas para o semiárido sergipano [37, 38, 39, 40]. No entanto, este resultado foi maior que o encontrado por Ruiz-Esparza (2014) [40], que verificou a percepção de agricultores no assentamento Jacaré-Curituba, Alto Sertão sergipano; e semelhante ao encontrado por SilvaAndrade et al. (2016) [17], que investigaram a percepção de agricultores convencionais e nãoconvencionais no semiárido pernambucano. Por outro lado, o número de espécies indicadas nesta pesquisa, mostra-se muito menor que o encontrado em outras pesquisas realizadas no semiárido Nordestino [41, 42].

Assim como no presente estudo, Silva-Andrade et al. (2016) [17] também constataram que as espécies de aves mais conhecidas pelos agricultores são aquelas mais comuns e abundantes em seus sistemas agrícolas. Os autores ainda demonstraram que as espécies mais conhecidas pelos agricultores são aquelas que também estão relacionadas com os conhecimentos que possuem sobre os usos para fins de alimentação, comércio e criação em gaiolas. Isso talvez explique porque as famílias Thraupidae, Columbidae e Tinamidae foram as mais representativas neste trabalho, pois espécies de aves dessas famílias (ex., Columbina talpacoti, Columbina picui, Crypturellus parvirostris e Paroaria dominicana) normalmente são mencionadas em vários trabalhos como animais de estimação e recurso alimentar [41, 43, 44, 45, 46].

Os produtores reconhecem que as aves visitam os pomares de goiaba em busca de alimento e local de repouso e nidificação, como evidenciado pelas respostas à pergunta 1 (As aves visitam as goiabeiras? O que elas fazem nas goiabeiras?). Todos responderam que as aves ocorrem nos pomares. A maioria (70\%) afirma que as aves visitam as goiabeiras para se alimentar das goiabas: "Elas visitam as goiabeiras pra comer as goiabas maduras" (Produtor 7); 19\% responderam comer goiabas e construir ninhos: "Vão atrás das goiabas maduras e de lugar pra fazer ninho" (Produtor 25); $6 \%$ comer goiabas e insetos: "Elas visitam pra comer as goiabas e as lagartas das goiabeiras" (Produtor 18); 3\% comer goiabas, insetos e fazer ninhos; e um único participante relatou que as aves visitam as goiabeiras para comer goiabas e repousar: "Visitam pra comer as goiabas madura e descansar" (Produtor 55).

Os frutos carnosos das goiabeiras são atrativos para as aves, especialmente para onívoros e frugívoros generalistas, que consomem seus frutos e, como observado em outro estudo [47], podem atuar na dispersão de sementes. Segundo Koopman e Pitt (2007) [48], algumas monoculturas criam condições favoráveis para algumas espécies de aves, em virtude, principalmente, da abundante oferta de alimento oferecida e, por isso, são frequentemente visitadas por aves e outros animais silvestres, sobretudo em períodos de escassez de alimento. Develey e Peres (2000) [49] ressaltam que variações na disponibilidade de recursos no período de seca, bem como o aumento da fragmentação e destruição de habitat, que comprometem a vegetação nativa, podem levar algumas espécies a buscarem fontes alternativas de alimento em áreas cultivadas.

Em um estudo conduzido por Mateus (2013) [50], em pomares comerciais de goiaba na Zona da Mata de Minas Gerais, também foi observado que algumas espécies de aves utilizaram a área tanto para eventos de alimentação quanto para nidificação. A autora identificou e confirmou a presença de sete espécies de aves que visitam os pomares em busca de alimento e seis espécies que os visitam para nidificar. Dentre as espécies observadas pela mesma autora, Tangara sayaca, Columbina talpacoti, Eupetomena macroura, Sporophila sp. e Sicalis flaveola podem ser observadas na lista de aves citadas pelos produtores de goiaba neste estudo. Campolim (2011) [51], observou sete espécies de aves utilizando pomares de tangerina no estado de São Paulo para nidificação, sendo Columbina talpacoti, Zenaida auriculata, Patagioenas picazuro, Eupetomena macroura e Pitangus sulphuratus comuns a este estudo.

Todos os produtores demonstraram que possuem apreço às aves, como evidenciado pela resposta à pergunta 3 (Você gosta de aves?). Assim, confirma a tendência relatada de que seres humanos e aves sempre mantiveram uma relação que envolve admiração e uso [52]. A maioria das respostas (59\%) foi justificada pelo apreço à beleza que as aves apresentam: "São bonitas. Tem umas que são bem coloridas" (Produtor 29); 21\% pelo apreço à beleza e ao canto: "São bonitas e eu gosto muito de ouvir elas cantando" (Produtor 5); 19\% pelo canto; e um único entrevistado afirmou gostar de aves pelo apreço à beleza e por servirem como alimento para a população humana: "Elas são bonitas e também são importantes pra nós, porque serve pra comer" (Produtor 21).

As motivações são também coincidentes visto que muitas espécies de aves são muito apreciadas pela beleza, mas especialmente pelo seu canto, sendo criadas como animais de estimação ou usadas 
pelas populações humanas na alimentação, o que estimula a caça direcionada a esse grupo de animais [53, 54], sobretudo no Nordeste brasileiro [41, 55, 56, 57, 58]. No Brasil, a caça é apontada como um dos crimes cometidos contra a fauna [59], e, como um tema polêmico, tem suscitado muitas discussões desde a publicação da Lei de Proteção à Fauna (Lei no 5.197/1967) [60]. A Lei de Crimes Ambientais (Lei $n^{\circ}$ 9.605/1998) [61], por exemplo, aventa, ainda que de maneira subjetiva, não ser crime o abate de um animal, quando realizado "em estado de necessidade, para saciar a fome do agente ou de sua família". Assim, a falta de uma definição legal e a dificuldade de compreensão do tema fazem com que as formas distintas de caça (ex., comercial, de controle, esportiva e de subsistência) sejam tratadas de maneira única [62].

A maioria dos entrevistados (69\% dos entrevistados) não reconhece as aves como elementos importantes dentro do ambiente agrícola. Para estes, as aves apenas causam prejuízos econômicos, como evidenciado pelas respostas à pergunta 4 (Você acha que as aves são importantes para a agricultura? Por quê?). Resultados semelhantes foram obtidos em outros estudos em áreas rurais, nos quais algumas espécies de aves não são apreciadas por agricultores, sobretudo por serem consideradas pragas agrícolas $[1,2,3,4]$. Fruticultores, por exemplo, não costumam tolerar a presença de algumas aves em seus sistemas de produção, pois muitas se alimentam dos frutos que são cultivados [3]. Isso também foi evidenciado nas respostas de alguns informantes: "Rapaz, eu acho que não. Se você for vê ali as goiaba, você vai encontrar um monte de goiaba bicada. Elas dão prejuízo pra gente" (Produtor 17); "Se não colher as goiaba madura logo, os passarinho bica tudo e não presta pra vender. Eles também fica procurando pra comer as sementes, quando a gente planta" (Produtor 13). Danos causados por aves às colheitas podem causar consideráveis perdas no rendimento em todo o mundo [63]. No entanto, poucos estudos quantificaram os danos causados por aves a culturas frutíferas [ver 2, 50, 64, 65].

Assim, pôde-se perceber que as interações negativas com as aves (danos às culturas) influenciam a percepção dos produtores sobre os possíveis benefícios fornecidos por esses animais aos sistemas de produção agrícola. Apenas $31 \%$ que responderam que as aves são importantes para os agroecossistemas, reconheceram a importância das aves como controle de pragas agrícolas, afirmando que as aves podem ser úteis na agricultura quando se alimentam de insetos: "Porque elas come os insetos e as lagarta que tem nas planta" (Produtor 3 ).

Alguns outros estudos [3, 15, 16, 17] que igualmente investigaram a percepção de produtores rurais em relação às aves também constataram que apenas um número reduzido de produtores reconhece alguns dos benefícios que as aves podem proporcionar às suas atividades agrícolas, como o controle de pragas, e que este serviço ecossistêmico prestado pode levar à redução no uso de agrotóxicos nas práticas agrícolas, sendo, portanto, útil para os agricultores. Neste sentido, o reconhecimento da importância das interações das aves com o ambiente agrícola precisa ser estimulado pelos que participam das atividades agrícolas [66, 67]. Jacobson et al. (2003) [15] e Herzon e Mikk (2007) [16] mostraram que um grande número de agricultores se mostrou disposto a conservar as espécies e, inclusive, atraí-las para as suas lavouras após conhecimento dos seus benefícios.

É expressivo o número de produtores (86\%) que que acham que as aves precisam ser protegidas (Pergunta 5). Desses, a maioria (66\%) justificou que as aves precisam ser protegidas porque são animais muito caçados: "Acho que sim, porque o povo caça demais pra criar em gaiola e comer" (Produtor 4); $13 \%$ declararam que as aves merecem proteção porque as matas estão sendo destruídas: "Porque estão acabando com as mata onde elas vive" (Produtor 33); 12\% porque fazem parte do patrimônio natural: "Porque são da natureza" (Produtor 45); 5\% por conta da caça e dos desmatamentos; e 3\% pelo apreço à beleza e ao canto: "Porque elas são bonitas e eu gosto do canto" (Produtor 2). Os entrevistados que responderam "Não" (14\%) alegaram que as aves não necessitam de proteção por serem abundantes na natureza: "Porque tem muita solta pelo mato. Esses bicho não acaba não, todo o ano tem" (Produtor 50).

A maioria dos produtores entrevistados reconhece que a caça e a perda de habitat, principais ameaças às aves brasileiras [68], afetam negativamente a avifauna. Das 510 espécies de aves registradas para o domínio da Caatinga [69], 20 (4\%) dessas já se encontram ameaçadas de extinção, devido, principalmente, às ações antrópicas que foram citadas por esses agricultores [70].

Quanto à pergunta 6 (Você faz algo para proteger as aves?), uma pequena parte dos entrevistados, representada por 16 informantes (19\%), respondeu "Sim", declarando que 
contribuem para a proteção das aves não praticando a caça, cuidando para que nenhuma fatalidade aconteça aos ninhos das espécies que nidificam em suas propriedades, e não utilizando defensivos químicos para o combate de pragas agrícolas: "Eu não caço, não pego pra criar e nem pra comer" (Produtor 26); "Eu deixo elas fazer ninho aqui nas planta à vontade. Não mexo e nem deixo ninguém mexer também" (Produtor 11); "Não passo veneno pra matar os insetos" (Produtor 9). Os que declararam "Não" (81\%), não justificaram o motivo de suas respostas.

Embora a maioria dos produtores $(86 \%)$ tenha respondido que as aves precisam ser protegidas, a maior parte também respondeu que não adotam qualquer atitude para contribuir com a proteção das aves. Apenas $19 \%$ dos entrevistados respondeu que protegem as aves não praticando a caça, protegendo os ninhos e não utilizando agrotóxicos. Assim, a agricultura desenvolvida no Nordeste brasileiro vem mantendo, de fato, sua função primordial de produção de alimentos, mas a conservação da biodiversidade, como das aves participantes de sistemas agrícolas, não vem sendo considerada como elemento importante a ser mantido nesses sistemas pelos agricultores, que normalmente não enxergam as aves como elementos importantes dentro do sistema agrícola [15, $16,17,50]$.

\section{CONCLUSÃO}

Foi constatado que a maioria dos produtores de goiaba do Alto Sertão sergipano conhecem poucas espécies de aves da região. Para estes produtores as aves não são importantes para a agricultura, mas apenas causadoras de prejuízos econômicos, por danificarem e se alimentarem dos frutos. Apesar disso, estes não utilizam práticas para afugentá-las ou reduzir as suas populações. Por outro lado, a falta de conhecimento sobre a função das aves como mediadoras de serviços ecossistêmicos relevantes para a agricultura (ex., controle de pragas) desestimulam atitudes voltadas à adoção de práticas para a atração ou manutenção das aves nos cultivos. A adoção de práticas agrícolas de baixo impacto, de modo a conciliar a produção agrícola com a conservação das aves, deve ser apoiada pelas políticas e ações conservacionistas em áreas agrícolas do semiárido, visto que estas promovem benefícios a múltiplos elementos da biodiversidade e para o bem-estar humano. Tais ações devem considerar a percepção do produtor sobre as aves e o sistema de produção local, bem como informações sobre os impactos da atividade agrícola sobre as populações de aves da região. Destaca-se, ainda, que a atuação dos produtores pode contribuir significativamente para amenizar impactos de atividades predatórias culturalmente adotadas como a caça e a criação de aves em cativeiro.

\section{AGRADECIMENTOS}

Os autores agradecem a Genivaldo A. de Jesus, Crislaine C. Calazans e Suelange O. Cruz pela ajuda nas coletas de dados em campo. A Daniela P. Bitencurti pela elaboração do mapa de localização das propriedades na área estudada. Aos produtores de goiaba do Alto Sertão sergipano, que se dispuseram a conceder as entrevistas e permitir a realização da pesquisa. Ao apoio financeiro da FAPITEC (edital: FAPITEC/SE/FUNTEC/CAPES N ${ }^{\circ}$ 07/2015 - LINHA 1 - Projeto de Pesquisa) e do CNPq/CAPES/INCT IN-TREE (chamada INCT - MCTI/CNPq/FAPs n ${ }^{\circ}$ 16/2014). O presente trabalho foi realizado com apoio da Coordenação de Aperfeiçoamento de Pessoal de Nível Superior - Brasil (CAPES) - Código de Financiamento 001.

\section{REFERÊNCIAS BIBLIOGRÁFICAS}

1. Gebhardt K, Anderson AM, Kirkpatrick KN, Shwiff SA. A review and synthesis of bird and rodent damage estimates to select California crops. Crop Prot. 2011 May;30:1109-1116, doi: 10.1016/j.cropro.2011.05.015

2. Kross SM, Tylianakis JM, Nelson XJ. Effects of introducing threatened falcons into vineyards on abundance of Passeriformes and bird damage to grapes. Conserv Biol. 2012 Feb;26:142-149, doi: 10.1111/j.1523-1739.2011.01756.x

3. Kross SM, Ingram KP, Long RF, Niles MT. Farmer perceptions and behaviors related to wildlife and onfarm conservation actions. Conserv Lett. 2018 Jan/Feb;11:1-9, doi: 10.1111/conl.12364

4. Pejchar L, Clough Y, Ekroos J, Nicholas KA, Olsson O, Ram D, Tschumi M, Smith HG. Net effects of birds in agroecosystems. BioSci. 2018 Nov;68:896-904, doi: 10.1093/biosci/biy104 
5. Schäckermann J, Weiss N, Von Wehrdren H, Klein AM. High trees increase sunflower seed predation by birds in agricultural landscape of Israel. Front Ecol Evol. 2014 Jul;2, doi: 10.3389/fevo.2014.00035

6. Galeotti $\mathrm{P}$, Inglisa M. Estimating predation impact on honeybees Apis mellifera L. by European bee-eaters Merops apiaster L. Rev Écol (Terre Vie). 2001;56:373-388.

7. Martin EA, Reineking B, Seo B, Steffan-Dewenter I. Natural enemy interactions constrain pest control in complex agricultural landscape. Proc Natl Acad Sci. 2013 Apr;110:5534-5539, doi: 10.1073/pnas. 1215725110

8. Dolbeer RA, Holler NR, Hawthorne DW. Identification and control of wildlife damage. In: Research and Management Techniques for Wildlife and Habitats; 1994; The Wildlife Society, Bethesda, Maryland, p. 474-506.

9. Lindell CA, Eaton RA, Lizotte EM, Rothwell NL. Bird consumption of sweet and tart cherries. HumanWildl Interact. 2012;6:283-290, doi: 10.26077/x5hh-j692

10. Mateus MB, Teixeira MCL, Ribon R. Situações de conflito entre produtores rurais e a fauna silvestre na Zona da Mata de Minas Gerais, Brasil, e sua opinião quanto ao uso da caça como ferramenta de controle. Biodivers Bras. 2018;8(2):251-266.

11. Anderson A, Lindell CA, Moxcey KM, Siemer WF, Linz GM, Curtis PD, Carroll JE, Burrows CL, Boulanger JR, Steensma KMM, Shwiff SA. Bird damage to select fruit crops: the cost of damage and the benefits of control in five states. Crop Prot. 2013 May;52:103-109, doi: 10.1016/j.cropro.2013.05.019

12. Dellamano F. Controlling birds with netting: blueberries, cherries and grapes. N Y Fruit Quart. 2006 Jun;14:3-5.

13. Whelan CJ, Wenny DG, Marquis RJ. Ecossystem services provided by birds. Ann N Y Acad Sci. 2008;1134:25-60, doi: 10.1196/annals. 1439.003

14. Sodhi NS, Sekercioglu CH, Barlow J, Robinson SK. Conservation of Tropical Birds. Oxford: John Wiley \& Sons. 2011.

15. Jacobson SK, Sieving KE, Jones GA, Van Doorn A. Assessment of farmer attitudes and behavioral intentions toward bird conservation on organic and conventional Florida farms. Conserv Biol. 2003 Apr;17(2):595-606, doi: 10.1046/j.1523-1739.2003.01472.x

16. Herzon I, Mikk M. Farmers' perceptions of biodiversity and their willingness to enhance it through agrienvironment schemes: A comparative study from Estonia and Finland. J Nat Conserv. 2007 Jan;15(1):10-25, doi: 10.1016/j.jnc.2006.08.001

17. Silva-Andrade HL, de Andrade LP, Muniz LS, Telino-Júnior WR, Albuquerque UP, Lyra-Neves RM. Do farmers using conventional and non-conventional systems of agriculture have diferente perceptions of the diversity of wild birds? Plos One. 2016 May;11(5):e0156307, doi: 10.1371/jornal.pone.0156307

18. Asokan S, Ali AMS, Manikannan R. Foraging behavior of selected birds in Cauvery Delta region of Nagapattinam District, Tamil Nadu, India. J Threat Taxa. 2010 Feb;2(2):690-694, doi: 10.11609/JoTT.o2201.690-4

19. Narayana BL, Pandiyan J, Rao VV. Studies on avian diversity in selected croplands of Nalgonda District, Andhra Pradesh, Southern India. In: Bhupathy S, Prusty BAK, Kumara HN, Jayaraj RSC, Quadros G, Pramod P, eds. Status of Indian Birds and Their Conservation. In Proceedings of "First International Conference on Indian Ornithology"; 2011; Coimbatore: Salim Ali Centre for Ornithology and Natural History (SACON), p. 47-49.

20. Narayana BL, Rao VV, Venkateswara Reddy V. Foraging behavior of Black drongo (Dicrurus macrocercus) in Nalgonda district of Andhra Pradesh, India. Bioscan. 2014 Jan;9(2):467-471.

21. Kellermann JL, Johnson MD, Stercho AM, Hackett SC. Ecological and economic services provided by birds on Jamaican Blue Mountain coffee farms. Conserv Biol. 2008 Oct; 22:1177-1185, doi: 10.1111/j.1523-1739.2008.00968.x

22. Mols CMM, Visser ME. Great tits (Parus major) reduce caterpillar damage in commercial apple orchards. Plos One. 2007 Feb;2:e202, doi: 10.1371/journal.pone.0000202

23. Haden VR, Niles MT, Lubell M, Perlman J, Jackson LE. Global and local concerns: What attitudes and beliefs motivate farmers to mitigate and adapt to climate change? Plos One. 2012 Dec;7:e52882, doi: 10.1371/journal.pone.0052882

24. Niles MT, Lubell M, Brown M. How limiting factors drive agricultural adaptation to climate change. Agric Ecosyst Environ. 2015 Feb;200:178-185, doi: 10.1016/j.agee.2014.11.010

25. Silva FO, Durigon A, Pereira AP, Silva C, Boscolo D, Calazans CC, Ruiz-Esparza DPB, Oliveira FA, Ribeiro GT, Matos JL, Ruiz-Esparza J, Barreto LMG, Cruz SO, Ribeiro Junior V. A biodiversidade que gera frutos no semiárido: o caso da goiabeira. 2019. Editora UFS: Universidade Federal de Sergipe.

26. IBGE. Instituto Brasileiro de Geografia e Estatística. 2018. Disponível em cidades.ibge.gov.br/brasil/se/pesquisa/15/11954?tipo=grafico\&indicador $=11956 \&$ ano $=2017$. Acesso 3 Junho 2019. 
27. Gomes CCS, Neto AOA, Barros AC, Lima CCV, Cunha LO. Perfil da produção agrícola no perímetro irrigado Califórnia-SE. Revista Verde. 2009 Jan/Mar;4(1):33-40.

28. CODEVASF. Companhia de Desenvolvimento dos Vales do São Francisco e do Parnaíba. Perímetros irrigados: Jacaré-Curituba. 2011. Disponível em codevasf.gov.br/linhas-de-negocio/irrigacao/projetospublicos-de-irrigacao/elenco-de-projetos/em-implantacao/jacare-curituba. Acesso 18 Junho 2019.

29. Albuquerque UP, Lucena RFP, Alencar NL. Métodos e técnicas para coleta de dados etnobiológicos. In: Albuquerque UP, Lucena RFP, Cunha LVFC, orgs. Métodos e técnicas na pesquisa etnobiológica e etnoecológica; 2010; Recife: Nupeea, p. 41-64.

30. Amorozo MCM, Viertler RB. A abordagem qualitativa na coleta e análise de dados em etnobiologia e etnoecologia. In: Albuquerque UP, Lucena RFP, Cunha LVFC, orgs. Métodos e técnicas na pesquisa etnobiológica e etnoecológica; 2010; Recife: Nupeea, p. 66-82.

31. Brasil. Resolução $n^{\circ} 466$, de 12 de Dezembro de 2012. Dispõe sobre diretrizes e normas regulamentadoras de pesquisas envolvendo seres humanos. Diário Oficial da República Federativa do Brasil, 16 Out. 1996. Disponível em http://bit.ly/1mTMIS3. Acesso 13 Julho 2020.

32. Medeiros PM, Almeida ALS, Lucena RFP, Souto FJB, Albuquerque UP. O uso de estímulos visuais na pesquisa etnobiológica. In: Albuquerque UP, Lucena RFP, Cunha LVFCC, orgs. Métodos e Técnicas na Pesquisa Etnobiológica e Etnoecológica; 2010; Recife: Comunigraf/Nupeea, p. 151-169.

33. Xeno-Canto. Compartilhando sons de aves do mundo todo. 2016. Disponível em xeno-canto.org. Acesso 22 Junho 2019.

34. Sigrist T. Guia de campo Avisbrasilis - Avifauna Brasileira: descrição das espécies. 2009. Vinhedo: Avisbrasilis.

35. Piacentini VQ, Aleixo A, Agne CE, Mauricio GN, Pacheco JF, Bravo GA, Brito GRR, Naka LN, Olmos F, Posso S, Silveira LF, Betini GS, Carrano E, Franz I, Lees AC, Lima LM, Pioli D, Schunck F, Amaral FR, Bencke GA, Cohn-Haft M, Figueiredo LFA, Straube FC, Cesari E. Annotated checklist of the birds of Brazil by the Brazilian Ornithological Records Committee / Lista comentada das aves do Brasil pelo Comitê Brasileiro de Registros Ornitológicos. Rev Bras Ornitol. 2015 Jun;23:91-298.

36. Silva C. Diversidade de aves silvestres e sua interação com pomares de goiaba (Psidium guajava L.) e produtores rurais no semiárido Nordestino [dissertação]. Sergipe (SE): Universidade Federal de Sergipe, 2019. $81 \mathrm{p}$.

37. Sousa MC. As aves de oito localidades do estado de Sergipe. Atual Ornitol. 2009 Mai/Jun;149:33-57.

38. Ruiz-Esparza JMA, Gouveia SF, Rocha PA, Beltrão-Mendes R, Ribeiro AS, Ferrari SF. Birds of Grota do Angico Monument in the semi-arid Caatinga scrublands of northeastern Brasil. Biota Neotrop. 2011 Apr/Jun; 11:269-276, doi: 10.1590/S1676-06032011000200027

39. Ruiz-Esparza JM, Rocha PA, Ribeiro AS, Ferrari AS. The birds of the Serra da Guia in the caatinga of northern Sergipe. Rev Bras Ornitol. 2012 Oct;20:290-301.

40. Ruiz-Esparza JMA. Sustentabilidade das comunidades de aves em duas áreas protegidas do estado de Sergipe [tese]. Sergipe (SE): Universidade Federal de Sergipe, 2014. 169 p.

41. Barbosa EDO, Silva MGB, Medeiros RO, Chaves MF. Atividades cinegéticas direcionadas à avifauna em áreas rurais do município de Jaçanã, Rio Grande do Norte, Brasil. Biotemas. 2014 Mai;27(3):175190, doi: 10.5007/2175-7925.2014v27n3p175

42. Pires-Santos D, Galvagne-Loss AT, Andrea MV, Costa-Neto EM. O conhecimento etnoornitológico dos moradores do município de Elísio Medrado, Bahia, Sergipe. Rev Ouric. 2015 Mar/Abr; 5(1):67-85.

43. Nobrega VA, Barbosa JAA, Alves RRN. Utilização de aves silvestres por moradores do município de Fagundes, Semiárido paraibano: uma abordagem etnoornitológica. Sitientibus. 2011 Ago;11(2):165-175.

44. Policarpo IS. Uso de aves silvestres no Brasil: aspectos etnozoológicos e conservação. [trabalho de conclusão de curso]. Paraíba (PB): Universidade Federal da Paraíba, 2013. 67 p.

45. Teixeira PHR. Conhecimento e uso efetivo da avifauna em uma comunidade no entorno da Floresta Nacional do Araripe - FLONA, Barbalha - CE [dissertação]. Pernambuco (PE): Universidade Federal Rural de Pernambuco, 2013. 53 p.

46. Galvagne-Loss AT, Costa-Neto EM, Flores FM. Aves silvestres utilizadas como recurso trófico pelos moradores do povoado Pedra Branca, Santa Terezinha, Bahia, Brasil. Gaia Scientia. 2014; Volume Especial Populações Tradicionais: 01-14.

47. Silva JCB, Cândido Júnior JF, Vogel HF, Campos JB. Dispersão por aves de Psidium guajava L. (Myrtaceae) em ambiente ripário na bacia do rio Paraná, Brasil. Semina. 2013 Jul/Dec;34(2):195-204.

48. Koopman ME, Pitt WC. Crop diversification leads to diverse bird problems in Hawaiian agriculture. Human-Wildl Conflicts. 2007;1(2):235-243.

49. Develey PF, Peres CA. Resource seasonality and the structure of mixed species birds flocks in a coastal Atlantic forest of southeastern Brazil. J Trop Ecol. 2000 Jan;16(1):33-53, doi: $10.1017 / \mathrm{S} 0266467400001255$ 
50. Mateus MB. Relação entre fauna silvestre e produtores rurais: estudos de caso em milho (Zea mays L.) e goiaba (Psidium guajava L.) na Zona da Mata, Minas Gerais [dissertação]. Minas Gerais (MG): Universidade Federal de Viçosa, 2013. 73 p.

51. Campolim MG. Utilização de sistemas agrícolas (tangerinas, Citrus reticulata) por aves na região de Pilar do Sul, São Paulo [dissertação] São Paulo (SP): Universidade Federal de São Carlos, 2011. 69 p.

52. Sick H. Ornitologia Brasileira. 1997. Rio de Janeiro: Nova Fronteira.

53. Rocha MSP, Cavalcanti PCM, Sousa RL, Alves RRN. Aspectos da comercialização ilegal de aves nas feiras livres de Campina Grande, Paraíba, Brasil. Rev Biol Ciênc Terra. 2006;6(2):204-221.

54. Trinca CT, Ferrari SF. Caça em assentamento rural na Amazônia Mato-grossense. In: Jacobi P, Ferreira LC, orgs. Diálogos em Ambiente e Sociedade no Brasil; 2006; São Paulo: Annablume, p. 155-167.

55. Alves RRN, Nogueira EG, Araújo HFP, Brooks SE. Bird-keeping in the Caatinga, NE Brazil. Hum Ecol. 2010 Feb;38(1):147-156, doi: 10.1007/s10745-009-9295-5

56. Bezerra DMM, Araújo HFP, Alves RRN. Captura de aves silvestres no semiárido brasileiro: técnicas cinegéticas e implicações para conservação. Trop Conserv Sci. 2012 Mar; 5(1):50-66.

57. Fernandes-Ferreira H, Mendonça SV, Albano C, Ferreira FS, Alves RRN. Hunting, use and conservation of birds in Northeast Brazil. Biodivers Conserv. 2012 Jan;21(1):221-244, doi: 10.1007/s10531-011-01799

58. Alves RRN, Leite RCL, Souto WMS, Bezerra DMM, Loures-Ribeiro A. Ethno-ornithology and conservation of wild birds in the semi-arid Caatinga of northeastern Brazil. J Ethnobiology Ethnomedicine. 2013 Feb;9(1):9-14, doi: 10.1186/1746-4269-9-14

59. Linacre A, Tobe SS. No overview to the investigative approach to species testing in wildlife forensic science. Investig Genet. 2011 Jan;2(2):2-9, doi: 10.1186/2041-2223-2-2

60. Brasil. Lei n ${ }^{\circ}$ 5.197, de 3 de Janeiro de 1967. Dispõe sobre a proteção à fauna e dá outras providências. Disponível em planalto.gov.br/ccivil_03/leis/L5197compilado.htm. Acesso 13 Julho 2020.

61. Brasil. Lei no 9.605, de 12 de Fevereiro de 1998. Dispõe sobre as sansões penais e administrativas derivadas de condutas e atividades lesivas ao meio ambiente, e dá outras providências. Disponível em planalto.gov.br/ccivil_03/leis/I9605.htm. Acesso 13 Julho 2020.

62. Novaro AJ, Redford KH, Bodmer RE. Effect of hunting in source-sink systems in the Neotropics. Conserv Biol. 2000 Jun;14(3):713-721, doi: 10.1046/j.1523-1739.2000.98452.x

63. De Grazio JW. World bird damage problems. In: Howard WE, Marsh RE, orgs. Proceedings of the 8th Vertebrate Pest Conference; 1978; University of California, Davis, Sacramento, CA.

64. Somers CM, Morris RD. Birds and wine grapes: foraging activity causes small-scale damage patterns in single vineyards. J Appl Ecol. 2002 Jun;39:511-523, doi: 10.1046/j.1365-2664.2002.00725.x

65. Mangan AM, Pejchar L, Werner SJ. Bird use of organic apple orchards: frugivory, pest control and implications for production. Plos One. 2017 Sep;12:1-15, doi: 10.1371/journal.pone.0183405

66. Costa-Neto EM, Santos Fita D, Vargas-Clavijo M. Manual de Etnozoología: una guia teórico-prática para investigar la interconexión del ser humano con los animales. 2009. Valencia: Tundra Ediciones.

67. McMahon JB, Anderson A, Carnus T, Helden AJ, Kelly-Quinn M, Maki A, Sheridan H, Purvis G. Different bioindicators measured at different spatial scales vary in their response to agricultural intensity. Ecol Indic. 2012 Jul;18:676-683, doi: 10.1016/j.ecolind.2012.01.013

68. Marini MA, Garcia FI. Conservação de aves no Brasil. Megadiversidade. 2005 Jul;1(1):95-102.

69. Silva JM, Souza MA, Bieber AGD, Carlos CJ. Aves da caatinga: status, uso do habitat e sensitividade. In: Leal IR, Tabarelli M, Silva JMC, orgs. Ecologia e Conservação da Caatinga; 2003; Recife: Editora Universitária da UFPE, p. 237- 273.

70. MMA. Ministério do Meio Ambiente. Livro Vermelho da Fauna Brasileira Ameaçada de Extinção. 2016. Disponível em mma.gov.br. Acesso em 20 Julho 2020. 